\title{
El lenguaje xenófobo en el discurso de los adolescentes en redes sociales. Análisis y propuestas de trabajo en el aula
}

\author{
Francisco José Sánchez García ${ }^{1}$
}

Recibido: 30 de noviembre de 2020 / Aceptado: 24 de abril de 2021

Resumen. El tratamiento en el aula del tema del racismo ha sido abordado extensamente en los últimos años, pero estimamos necesaria una revisión exhaustiva del papel de las redes sociales en su reproducción discursiva entre los adolescentes españoles. Como demuestran estudios recientes, el auge del sentimiento nacionalista/patriótico español discurre en paralelo al crecimiento de fake news sobre migrantes y los mensajes xenófobos en redes sociales, lo cual es especialmente preocupante en el caso de los menores, mucho más permeables a la manipulación. En este artículo se constata la necesidad de dar cabida en el currículo a la educación en valores, implementando iniciativas didácticas que aborden específicamente el discurso del odio a personas de otras razas o procedentes de otros países. Proponemos recurrir a la red social Twitter para trabajar en clase con textos reales acerca de la cuestión migratoria, a fin de estimular el debate y la participación activa de los estudiantes en su proceso de aprendizaje, guiándolos en la detección de noticias falsas y mensajes discriminatorios, de modo que puedan disponer de las herramientas necesarias para construir su identidad en un marco de respeto, inclusión y tolerancia.

Palabras clave: Discurso del odio; discurso xenófobo; racismo; innovación educativa; redes sociales.

[en] The xenophobic language in the speech of teenagers on social networks. Analysis and work proposals in the Secondary Classroom

\begin{abstract}
The issue of racism in the classroom has been extensively addressed in recent years, but we consider that an exhaustive review of the role of social networks in its discursive reproduction among Spanish adolescents is necessary. As recent studies show, the rise of Spanish nationalist / patriotic sentiment runs parallel to the growth of fake news about migrants and xenophobic messages on social networks, which is especially worrying in the case of minors, who are much more permeable to manipulation. This article confirms the need to give room to values education in the classroom, implementing educational initiatives that specifically address hate speech to people of other races or from other countries. We propose to use the social network Twitter to work in class with real speeches about the immigration issue, in order to stimulate debate and the active participation of students in their learning process, guiding them in the detection of false news and discriminatory messages, so that they can have the necessary tools to build their identity within a framework of respect, inclusion and tolerance.
\end{abstract}

Keywords: Hate speech; xenophobic speech; racism; educational innovation; social networks.

[fr] La langue xénophobique dans le discours de adolescents sur les réseaux sociaux. Analyse et propositions de travail en classe

Résumé. Le traitement du thème du racisme en classe a été largement abordé ces dernières années, mais nous considérons qu'une revue exhaustive du rôle des réseaux sociaux dans sa reproduction discursive chez les adolescents espagnols est nécessaire. Comme le montrent des études récentes, la montée du sentiment nationaliste / patriotique espagnol est parallèle à la croissance des fake news sur les migrants et des messages xénophobes sur les réseaux sociaux, ce qui est particulièrement inquiétant dans le cas des mineurs, beaucoup plus perméables à la manipulation. Cet article confirme la nécessité d'inclure l'éducation aux valeurs dans le programme, en mettant en œuvre des initiatives éducatives qui abordent spécifiquement le discours de haine contre des personnes d'autres races ou d'autres pays. Nous proposons d'utiliser le réseau social Twitter pour travailler en classe avec de vrais textes sur la question de l'immigration, afin de stimuler le débat et la participation active des étudiants dans leur processus d'apprentissage, en les guidant dans la détection des fausses nouvelles et des messages discriminatoires, afin qu'ils disposent des outils nécessaires pour construire leur identité dans un cadre de respect, d'inclusion et de tolérance.

Mots-clés: Discours de haine; discours xénophobe; racisme; innovation pédagogique; médias sociaux.

Sumario: 1. Introducción. 2. La percepción social de la inmigración entre la juventud española. 3. El tratamiento del discurso xenófobo en el aula de Secundaria. 3.1. Retos para los docentes del s. XXI. Hacia una educación inclusiva. 3.2. El discurso del odio en las redes. Propuestas de implementación didáctica. 4. Conclusiones. 5. Bibliografía

Cómo citar: Sánchez García, Francisco José (2021). El lenguaje xenófobo en el discurso de los adolescentes en redes sociales. Análisis y propuestas de trabajo en el aula. Didáctica. Lengua y Literatura, 33, 121-131.

Departamento de Lengua Española, Universidad de Granada.

franjose@ugr.es 


\section{INTRODUCCIÓN}

Como resultado de la diversidad cultural, en las últimas décadas hemos asistido a una rápida transformación del tejido social español. Es evidente que la realidad cambiante de nuestro país exige una educación comprometida con el hecho intercultural. El punto de partida ineludible pasa por concebir la enseñanza desde una perspectiva transversal en la que se prime la formación inclusiva del alumnado. Pero entendemos que una educación intercultural basada en valores que aspire a resultar verdaderamente útil no puede quedarse en una mera declaración de intenciones (Jiménez Mañas, 2012, p. 99). Se trata de poner el foco de atención en la manera en que se originan y reproducen los mensajes discriminatorios, especialmente a través de las vías de comunicación preferidas por los jóvenes.

Son muchas las razones que nos llevan a plantear la necesidad de incorporar en el currículo de Secundaria y Bachillerato el fenómeno del discurso populista en redes sociales. En una sociedad cambiante y global, en la que la juventud se ve a diario bombardeada por un sinfín de mensajes sin contrastar, es peligrosamente fácil que se deslicen discursos de intolerancia y discriminación. Por tanto, no solo nos interesa llamar la atención en clase sobre los conceptos de xenofobia o el racismo, sino proponer como ejemplos textos de jóvenes del rango de edad de los estudiantes difundidos en las redes, como forma más eficaz para desmontar los argumentos populistas que perciben la alteridad como amenaza.

Indudablemente, el análisis del papel de la prensa tradicional y los nuevos medios interactivos es especialmente relevante en el contexto actual. Entre los estudios dedicados a analizar la creación, difusión y reproducción discursiva de los mensajes racistas a través de los medios de comunicación, indudablemente, una de las aportaciones más destacadas es la del lingüista holandés Teun A. van Dijk (1997), centrada en el examen crítico del tratamiento de las minorías étnicas en la prensa escrita y las estrategias ideológicas vehiculadas a través de los titulares y los artículos de opinión.

La necesidad de dicho análisis cobra especial importancia si tenemos en cuenta que, en los últimos años, en los países europeos asistimos al surgimiento de nuevos partidos populistas de corte conservador o ultraliberal, como Amanecer Dorado en Grecia, el Frente Nacional en Francia o Vox en España, caracterizados por lanzar mensajes de fuerte sentimiento identitario patriótico/nacionalista y, por ende, muy agresivos contra la inmigración ilegal o la integración social de los extranjeros en nuestro país (acceso a los servicios públicos, por ejemplo). En paralelo, la universalización del acceso a Internet ha traído consigo una multiplicación exponencial de mensajes de carácter excluyente en las redes sociales, lo cual se está convirtiendo en un preocupante fenómeno de reproducción ideológica del discurso xenófobo de extrema derecha, que llega ahora a personas a priori no necesariamente alineadas con una ideología radical.

Poco a poco, esta emergencia se va traduciendo en datos tangibles muy preocupantes, de indudable impacto social. De acuerdo con el informe de 2019 emitido por el Ministerio del Interior sobre los delitos de odio cometidos en España, los casos ascendieron a 1706 en 2019, un 6,8\% más que en 2018. En cuanto a los delitos registrados, un total de 194 personas fueron detenidas o investigadas por racismo o xenofobia, siendo la causa que más aumenta (un 20,9\%), por encima de la discriminación por ideología, identidad sexual o de género, religión o aporofobia. Por último, si nos fijamos en el medio de difusión, llama la atención el incremento de casos de delitos de odio cometidos a través de Internet (lo que conocemos como web 1.0) y redes sociales (web 2.0, atendiendo a su potencial interactivo) en un solo año: un 49,4\% con respecto a 2018, siendo las amenazas o las injurias los tipos de hecho más destacados. La comisión de estos delitos se sirve de Internet como medio preferente $(54,9 \%)$ si bien las redes sociales ganan terreno progresivamente, situándose como el segundo medio empleado:

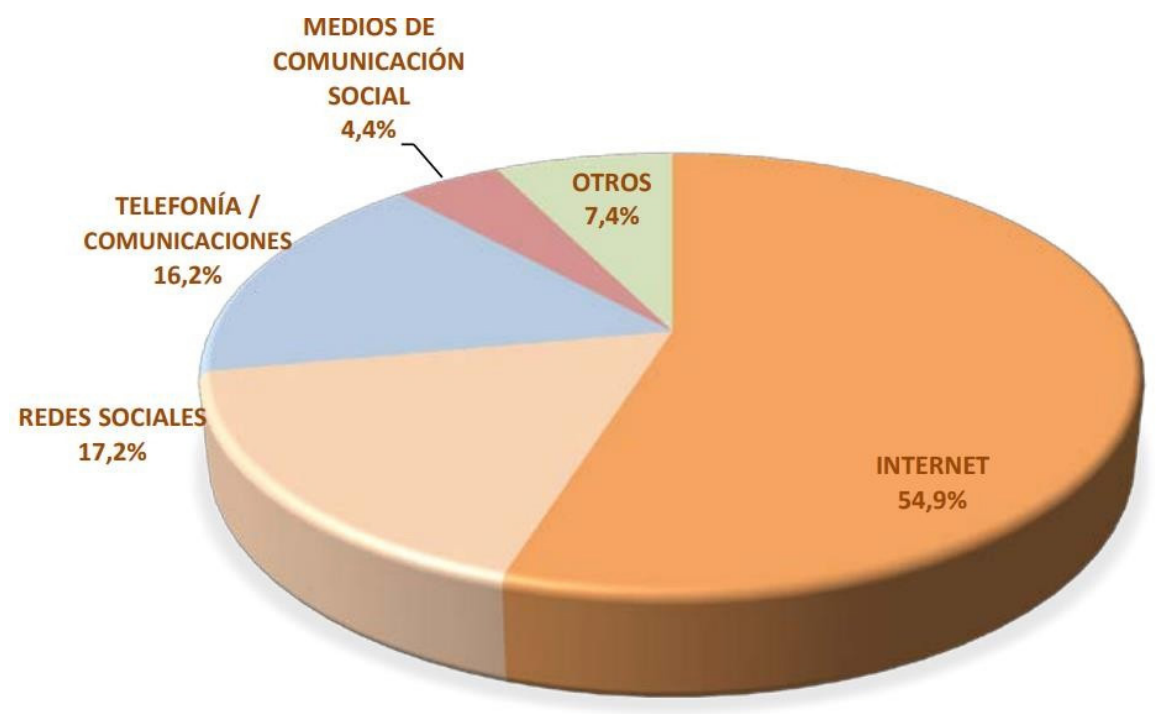

Figura 1. Delitos de odio cometidos a través de Internet y redes sociales. Fuente: Ministerio del Interior (2019). 
Este documento no refleja el dato desagregado por comunidades autónomas, que sí aparece recogido en el Informe anual de "SOS Racismo" para el estado español (2018, p. 5), documentándose un mayor número de casos en Aragón (36,2\%), Cataluña (25,3\%) y Galicia (19,5\%). Nótese, no obstante, que estos datos no son estrictamente comparables con los que ofrece el Ministerio del Interior, pues aquí se indican denuncias de discriminación que abarcan conflictos y agresiones racistas, denegación de acceso a prestaciones, discriminación laboral, discurso del odio de la extrema derecha, racismo institucional y abusos de las fuerzas de seguridad, entre otros.

En el ámbito específico de la educación, estudios recientes sobre la convivencia en las aulas españolas entre alumnos nacionales y los de origen extranjero evidencian que las conductas de discriminación en el ámbito escolar son un problema relevante: nada menos que un 57\% de los profesores encuestados por Peiró i Gregori y Merma (2012) afirman haber tenido que intervenir frente a este tipo de conductas, de los cuales, cerca de un $40 \%$ se encuadrarían claramente como xenofobia o racismo. Estas actitudes, basadas en prejuicios hondamente arraigados en algunos jóvenes, exigen una reflexión seria sobre las razones socio-educativas que explican el fenómeno y el modo en que se concretan en sus manifestaciones discursivas.

\section{LA PERCEPCIÓN SOCIAL DE LA INMIGRACIÓN ENTRE LA JUVENTUD ESPAÑOLA}

Para empezar, conviene que nos detengamos a examinar la percepción que tienen las nuevas generaciones acerca de los inmigrantes. Debemos partir de un concepto clave, el de identidad cultural, tratado extensamente por O’Connor y Faas (2012), Steinbhach (2014) o Escalona Velázquez (2012). Como nos recuerdan Cobano y Llorent (2017), la identidad cultural es un proceso dinámico construido individualmente, pero también colectivamente, en tanto que se retroalimenta de las influencias del entorno. Pensando en el rango de edad de los adolescentes, parece claro que su proceso de adquisición de la identidad cultural surge de la autovaloración en relación a los "otros", de modo que para comprender esa identidad "debemos tener en cuenta también la ajena" (Cobano y Llorent, 2017, p. 84).

En estudios dedicados específicamente a la percepción de la inmigración por parte de los jóvenes, como los de Jiménez Bautista (2005 y 2006), llama la atención que los principales problemas para los adolescentes son la inseguridad, el desempleo y los inmigrantes. De hecho, se presenta como dato destacable que vinculen los tres problemas como uno solo. En el discurso racista de algunos adolescentes, este concepto de alteridad se asienta sobre una serie de marcos conceptuales mediante los cuales se propende a identificar a los "otros" como una amenaza latente, un colectivo hostil que persigue apropiarse de los logros y derechos alcanzados gracias al estado del bienestar. En efecto, la imagen del "otro" va intrínsecamente ligada a 'problema', 'conflicto', 'delincuencia'. En una sociedad desinformada - paradójicamente, a causa del exceso de información, lo que Castells (1999) denomina "sobreinformación”cala cada vez más el discurso que construye la imagen del "otro" como nuestro enemigo, el antagonista natural de Occidente. Como señala Jiménez Bautista (2006, p. 563 y ss.), la construcción arquetípica de esa imagen de los otros se concreta en los valores de nacionalidad, raza, religión, cultura y clase social:

En primer lugar, hay que destacar cómo los jóvenes tienen una imagen muy clara sobre la nacionalidad de ese otro, de forma que, de cada diez jóvenes de la ciudad de Granada, nueve saben que cuando piensan en otra nacionalidad están pensando en los norteafricanos y los centroafricanos (...). En segundo lugar, la raza nos va a señalar la unión especial que puede existir entre esa construcción social y el color, a la hora de ser percibidos por parte de los jóvenes de Granada. Se observa que el color predominante es el color negro (...). En tercer lugar, la religión nos va a indicar cuál es la religión más importante en ese otro. Podemos señalar cómo sigue siendo predominante el dominio de la religión musulmana (...) En cuarto lugar, llama la atención el predominio de la cultura musulmana (...) En quinto lugar, se pregunta sobre la clase social, donde existe una bipolaridad entre la clase alta y clase baja, de forma que la clase baja gana de forma considerable a la clase alta. Además, hay que incluir los excluidos que suelen ser señalados por parte de los jóvenes con unos porcentajes considerables y de igual forma la variable gitano como un elemento de diferenciación social dentro del colectivo de jóvenes.

Esto lleva al antropólogo Jiménez Bautista a concluir que el racismo y la xenofobia “están impregnados de forma estructural en los jóvenes de Granada" (2006, p. 573). La conclusión del autor no es precisamente muy alentadora, al entender que "si la presencia de estos grupos de inmigrantes y la presencia de clases sociales más desfavorecidas fuese mayor, la tendencia social sería la de una actitud y un comportamiento abiertamente discriminatorio, racista y xenófobo" (2006, p. 575).

En otras investigaciones se llega a conclusiones análogas, como el estudio de Mata y Ballesteros (2008), o el de Calvo Buezas (2012) sobre los jóvenes extremeños:

Es muy amplia la complejidad de factores que en ella inciden, pero siempre presenta una actitud de aversión a los venidos de fuera, sentada en el sentimiento de la propia superioridad, la posesión excluyente de la tierra, el deseo de autoafirmación enfrentada, el eurocentrismo, con todas sus formas culturales, sociales y religiosas, y 
el recurso a un chivo expiatorio sobre el que proyectar la responsabilidad de los propios males para defenderse de los que consideran enemigos; todo lo cual genera y alimenta el fanatismo en dominar, explotar y marginar a los demás. (2012, p. 14).

A grandes rasgos, los jóvenes españoles que "compran” el discurso populista xenófobo, enmarcan la problemática de la inmigración en un manojo de frames muy reconocibles:

a. Los extranjeros crean problemas.

b. Los extranjeros nos quitan el trabajo (no hay trabajo para todos).

c. Los extranjeros son delincuentes.

d. Los extranjeros no se adaptan a nuestra cultura.

Los datos de este trabajo revelan además un emergente (y alarmante) cariz violento en la expresión de rechazo al extranjero. En algunos casos, estas actitudes excluyentes están correlacionadas con un fuerte sentimiento patriótico o nacionalista ("fuera los extranjeros/ ¡Viva España!”). A la luz de estudios como los anteriormente expuestos, parece necesario incorporar al currículo de Secundaria y Bachillerato unidades didácticas -en todas las materias, pero especialmente en Lengua Castellana y Literatura- específicamente dedicadas a la desmitificación de ciertos estereotipos xenófobos y racistas, toda vez que determinadas ideologías populistas parecen estar calando en la juventud española, especialmente desde la aparición del partido Vox.

No podemos perder de vista que los nacidos en el s. XXI, en tanto que "nativos digitales" (Prensky, 2001) no se informan a través de los medios de comunicación tradicionales, como puede ser la prensa en papel (ni siquiera digital) o los noticiarios de radio y televisión: directamente construyen su imaginario de la actualidad a través de inputs virales que les llegan a su teléfono móvil a través de aplicaciones de mensajería y redes sociales. Huelga decir que esta suerte de "pseudonoticias" carecen de rigor informativo, y su fiabilidad debería ser puesta en cuarentena por cualquier lector crítico, pues en su mayoría obedecen a estrategias de clickbait, por las cuales se persigue obtener el mayor número de visitas posible a una web, lo cual se consigue recurriendo a titulares tendenciosos o escandalosos, que apelan directamente a los miedos o temores de los ciudadanos. En el caso que nos ocupa, la mayoría de "noticias virales" vinculadas a la inmigración están correlacionadas con la delincuencia, la marginalidad o el crimen organizado.

\section{EL TRATAMIENTO DEL DISCURSO XENÓFOBO EN EL AULA DE SECUNDARIA}

\subsection{Retos para los docentes del s. XXI. Hacia una educación inclusiva}

A tenor de lo expuesto, pensamos que resulta muy necesario visibilizar en el aula el problema de la xenofobia y la discriminación, sensibilizando a los estudiantes sobre las consecuencias del discurso racista y eurocéntrico y llamando la atención sobre los contenidos virales de Twitter o WhatsApp. Es fundamental dar cabida al compromiso ético en el currículo de Secundaria y Bachillerato, para que los discentes tomen conciencia del enriquecimiento que supone vivir en una sociedad diversa e inclusiva, y sean capaces de combatir argumentativamente las noticias populistas de corte marcadamente discriminatorio.

Coincidimos con Jiménez Mañas (2012) en que:

Sin conocimiento, se anulan las posibilidades de elección y por tanto de toma de decisiones; por lo tanto, los contenidos forman en la educación ética un todo inseparable y entre ellos se ha de incluir, de forma intencional, ideas o hechos que inciten a la discusión, que provoquen debates grupales, para que los alumnos extraigan sus propias perspectivas, compartan o difieran, tomen conciencia de pertenencia a un grupo $\mathrm{y}$ adquieran las herramientas necesarias como personas, como parte de un grupo, como miembros de una sociedad (p. 99).

Por supuesto, en los últimos años, la normativa se ha ido adaptando a esta problemática. La LOMCE, en su artículo 123, resalta la importancia de incentivar el pensamiento crítico:

Las habilidades cognitivas, siendo imprescindibles, no son suficientes; es necesario adquirir desde edades tempranas competencias transversales, como el pensamiento crítico, la gestión de la diversidad, la creatividad o la capacidad de comunicar, y actitudes clave como la confianza individual, el entusiasmo, la constancia y la aceptación del cambio. La educación inicial es cada vez más determinante por cuanto hoy en día el proceso de aprendizaje no se termina en el sistema educativo, sino que se proyecta a lo largo de toda la vida de la persona (2013, p. 5)

En este sentido, la LOMCE refleja la necesidad de garantizar la inclusión educativa y de favorecer la resolución pacífica de los conflictos que se producen en la realidad de las aulas. No obstante, debemos decir que el hecho de 
que quede recogido en la normativa de esta manera tan indirecta no supone que para los docentes resulte fácil implementarlo habida cuenta de la radicalización de algunos estudiantes, especialmente en los centros calificados como de "difícil desempeño".

Esta circunstancia obliga a los docentes a buscar estrategias constantemente para trasladar este tema al aula, procurando llevar a cabo intervenciones educativas basadas en los principios de significatividad — que priorice una implicación activa y responsable de los estudiantes en su aprendizaje, fomentando la participación-, diálogo — mediante la exposición y confrontación de sus opiniones y puntos de vista- y autoconocimiento - el intercambio no solo les permitirá conocerse mejor, sino también reconocer sutiles actitudes de discriminación de las que no son plenamente conscientes- (Puig Rovira y Martín García, 1998).

No podemos perder de vista el carácter dinámico y maleable del concepto de identidad cultural en la adolescencia, que nos permite "transformarnos y enriquecernos a través del contacto con otras culturas, pudiendo llegar a realizar pequeños cambios que modifiquen nuestra identidad cultural, o grandes cambios que lleguen a crear nuevas identidades" (Escalona Velázquez, 2012, p. 4).

Educar en valores pasa también por entender el aula como un espacio de educación intercultural inclusiva. Naturalmente, ello conlleva un cambio de mentalidad en buena parte del personal docente, que deberá replantear su modelo de enseñanza para implicar a los discentes. Como señalan Terrón, Cárdenas y Rodríguez (2017, p. 27), “la transformación se consigue, en gran medida, desde abajo, desde la implicación de los/las protagonistas clave que participan en los procesos de enseñanza-aprendizaje, así como al afirmar que el papel del/la docente es muy importante en dicho proceso". El desempeño de este papel crucial coloca a los docentes ante uno de los mayores retos de la educación. Como señala Bauman:

El cambio actual no es como los cambios del pasado. En ningún otro punto de inflexión de la historia humana los educadores debieron afrontar un desafío estrictamente comparable con el que nos presenta la divisoria de aguas contemporánea. Sencillamente nunca antes estuvimos en una situación semejante. Aún debemos aprender el arte de vivir en un mundo sobresaturado de información. Y también debemos aprender el aún más difícil arte de preparar a las nuevas generaciones para vivir en semejante mundo. (Bauman, 2008, p. 46)

¿Cómo lograr la ansiada implicación de los estudiantes en el proceso de enseñanza-aprendizaje de un tema tan sensible? Machado Casas, Alanis y Ruiz (2017) destacan la trascendental relevancia de las TIC (Martínez Ezquerro, 2016) como herramienta de inclusión educativa en la diversidad cultural.

Por supuesto, en tanto que "nativos digitales" (Prensky, 2001), los adolescentes están conectados permanentemente a las pantallas, y viven su vida a través de las redes sociales, aunque, a tenor de los datos, resulta evidente que no son del todo conscientes de sus peligros. Porque sabemos que no todos los contenidos que nos llegan a través de Internet son rigurosos o fiables, y, por ende, las nuevas generaciones han de recibir un adiestramiento específico en el manejo de las herramientas digitales, una suerte de "alfabetización digital" (Brites, McDougall, Couto y Lucas, 2019) o "mediática” (Fernández García, 2017) que les ayude a desenvolverse en los nuevos medios con plenas garantías.

Resulta fundamental que los docentes actualicen sus conocimientos de la mano de proyectos nacionales e internacionales, para poder implementar en el aula actividades que puedan ser eficaces y fructíferas. Un buen ejemplo lo encontramos en el proyecto colaborativo "CibeRespect" (2018), desarrollado por Durán, Sueiro y García como plataforma para monitorizar estos mensajes de intolerancia racial o cultural que se amplifican y difunden de manera exponencial a través de Internet. Las propias autoras destacan algunos precedentes de iniciativas encaminadas a abordar la problemática del discurso racista, entre las que destacan las campañas de sensibilización cultural llevadas a cabo por organizaciones como Intermón-Oxfam, acciones de sensibilización para periodistas, o las llamadas "redes anti-rumores" puestas en marcha por el Gobierno Vasco, la Junta de Andalucía, la Generalitat de Cataluña o la Comunidad de Madrid para combatir la difusión de estereotipos negativos sobre los migrantes.

Destacamos también el proyecto coordinado por Olmos Alcaraz "Jóvenes, redes virtuales y nuevas lógicas de funcionamiento del racismo" (2017). Este trabajo profundiza en un campo de estudio aún inexplorado: el análisis de las lógicas de funcionamiento del racismo en nuestra era virtual, evaluando su impacto, influencia y potencial función de predicción de comportamientos sociales.

\subsection{El discurso del odio en las redes. Propuestas de implementación didáctica}

Como antes indicábamos, pensamos que Twitter puede ser una buena herramienta para mostrar en clase el potencial persuasivo de determinados mensajes populistas de corte xenófobo o racista. La red de microblogging es el paradigma de lo que hoy entendemos como Web 2.0, caracterizada por el intercambio de conocimiento e información a través de Internet, y, en consecuencia, convierte al usuario de Internet en creador y difusor de contenidos. De esta nueva dinámica emerge un nuevo discurso que participa de esta naturaleza interactiva que nos brinda la red, en la medida en que permite el intercambio comunicativo entre usuarios que no se conocen, pero que comparten intereses 
comunes. Esta mecánica de interacción contribuye a reafirmar la identidad individual, tanto cuando los usuarios se muestran a favor de los argumentos o noticias difundidas, como cuando, por el contrario, se intensifica la polarización al manifestarse opiniones radicalmente opuestas. En la práctica, podríamos pensar que esta nueva forma de opinión virtual (e-democracy) representa una enorme ventaja, ya que está sirviendo para universalizar la participación en el debate público de manera exponencial (Iyengar, Luskin y Fishkin, 2004). También hay quienes ven aquí una suerte de "barómetro social" (Congosto, 2014), que nos brinda la oportunidad de examinar fácilmente la génesis y difusión de corrientes de pensamiento.

Como sabemos, Twitter se sitúa a mitad de camino entre el blog y la red social, siendo una de sus características más sobresalientes la limitación de caracteres de las entradas (originalmente, eran 140 caracteres), dando como resultado una comunicación asimétrica en la que el emisor puede filtrar los comentarios que recibe cada una de sus entradas en la red (Mancera y Pano, 2013). Se supone que la plataforma está pensada para incentivar la conversación (no solo permanecer informados o "seguir" a emisores erigidos en referentes), y aunque son habituales los debates (y célebres las polémicas entre miembros conocidos de la red) en la práctica, los usuarios acaban limitando su interacción con aquellos que piensan y se expresan como ellos.

Los partidos políticos, y especialmente las nuevas formaciones, obligadas a posicionarse en la escena pública para dar a conocer su ideario entre sus potenciales seguidores, se sirven de Twitter para lanzar mensajes y tratar de dirigir el debate público según sus intereses. Esta forma de comunicación pone en marcha procesos de construcción identitaria (aunque se trate de una forma simplificada, casi esquemática, de las identidades), como señalan Olmos et al. (2020).

Los docentes pueden aprovechar el potencial que les brinda la red para analizar los mensajes políticos que, para el caso que nos ocupa, den lugar a manifestaciones que podríamos etiquetar como "racistas" o "xenófobas".

Por ejemplo, analicemos el siguiente hilo iniciado por la cuenta oficial del partido de extrema derecha española "Vox". Como puede verse en la figura 2, se resume en un tuit un manojo de datos una nota de prensa del partido, en el que se establece la asociación directa entre la llegada de inmigrantes ilegales a las costas españolas y la culpabilidad del gobierno socialista (queda explícitamente expresado en el hashtag "Gobierno culpable del \#EfectoInvasion").

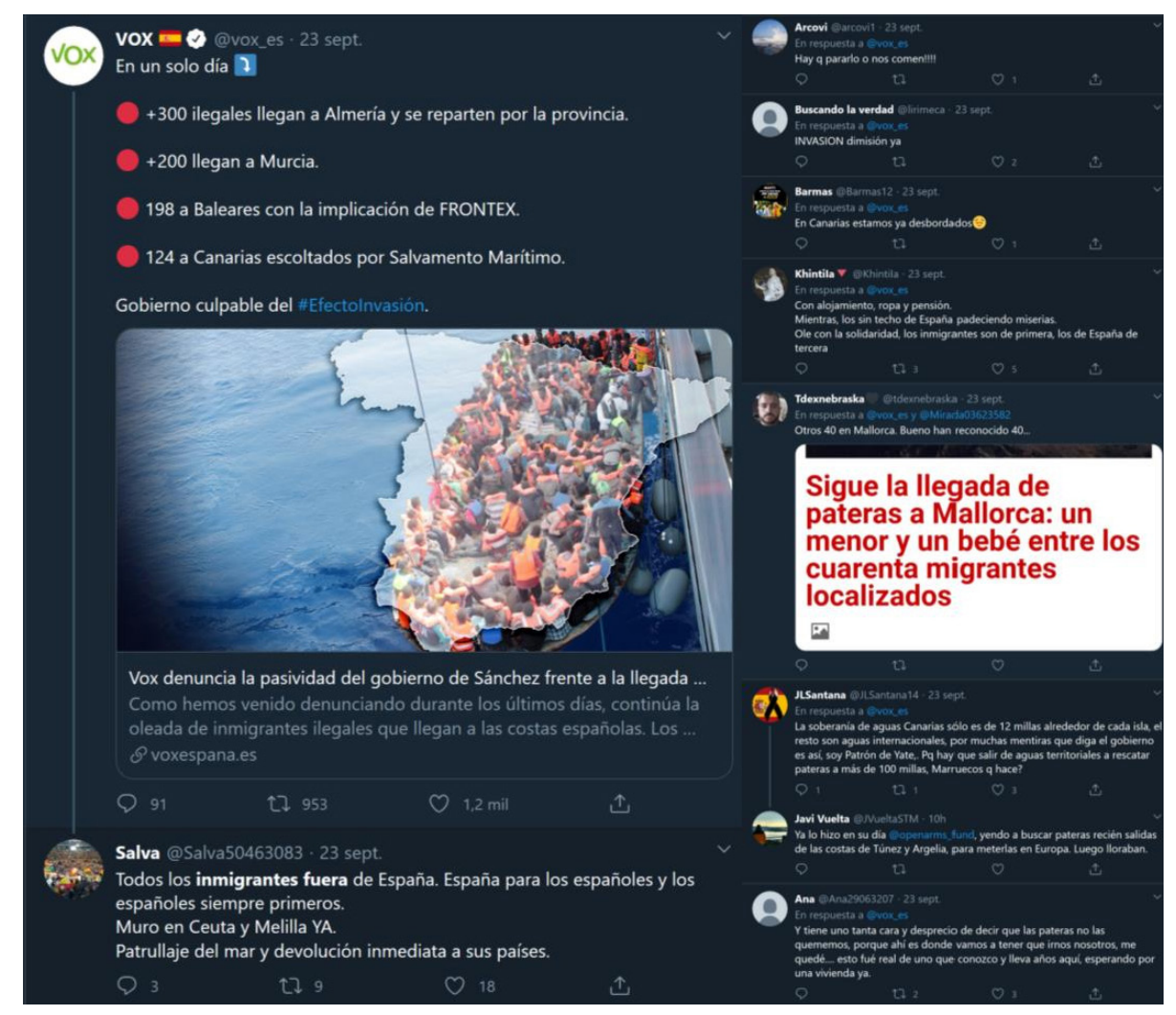

Figura 2. Hilo de Twitter en respuesta a una entrada del partido Vox (23/9/2020). Fuente: captura de pantalla de la red social Twitter.

Sin duda, tan interesante como el propio tuit son las respuestas de los seguidores de Vox en Internet. Una actividad sencilla y estimulante para trabajar en clase es el estudio léxico de las respuestas a este tipo de consignas discriminatorias. En nuestro ejemplo, basta con copiar el texto con las respuestas del hilo completo del tuit de Vox y crear una "nube de tags", que consiste en un mosaico formado por las palabras más 
repetidas. Para ello, podemos utilizar páginas web de acceso libre como WordClouds, TagCrownd o Wordle, entre otras. La ventaja de esta herramienta es que obtenemos una "radiografía" sumamente ilustrativa (y muy atractiva para su manejo en el aula) de los campos semánticos más frecuentes de un conjunto textual de un modo rápido y sencillo. Del "generador de nubes de palabras" resulta una imagen formada por los términos más destacados, que aparecen distribuidos en una imagen, en diferentes tamaños y colores (de mayor a menor importancia, según su colocación central o marginal y su tamaño relativo). En la figura 3 tenemos la nube de tags generada a partir del tuit de Vox que venimos comentando. Como puede apreciarse, las palabras más llamativas (y, por ende, las más repetidas por los usuarios que han comentado la entrada de Vox) son España, expulsión, invasión, pateras, ilegales... De un solo golpe de vista, nos hacemos una idea bastante precisa de los marcos conceptuales presentes en el hilo, quedando de manifiesto el sesgo claramente discriminatorio hacia los migrantes.

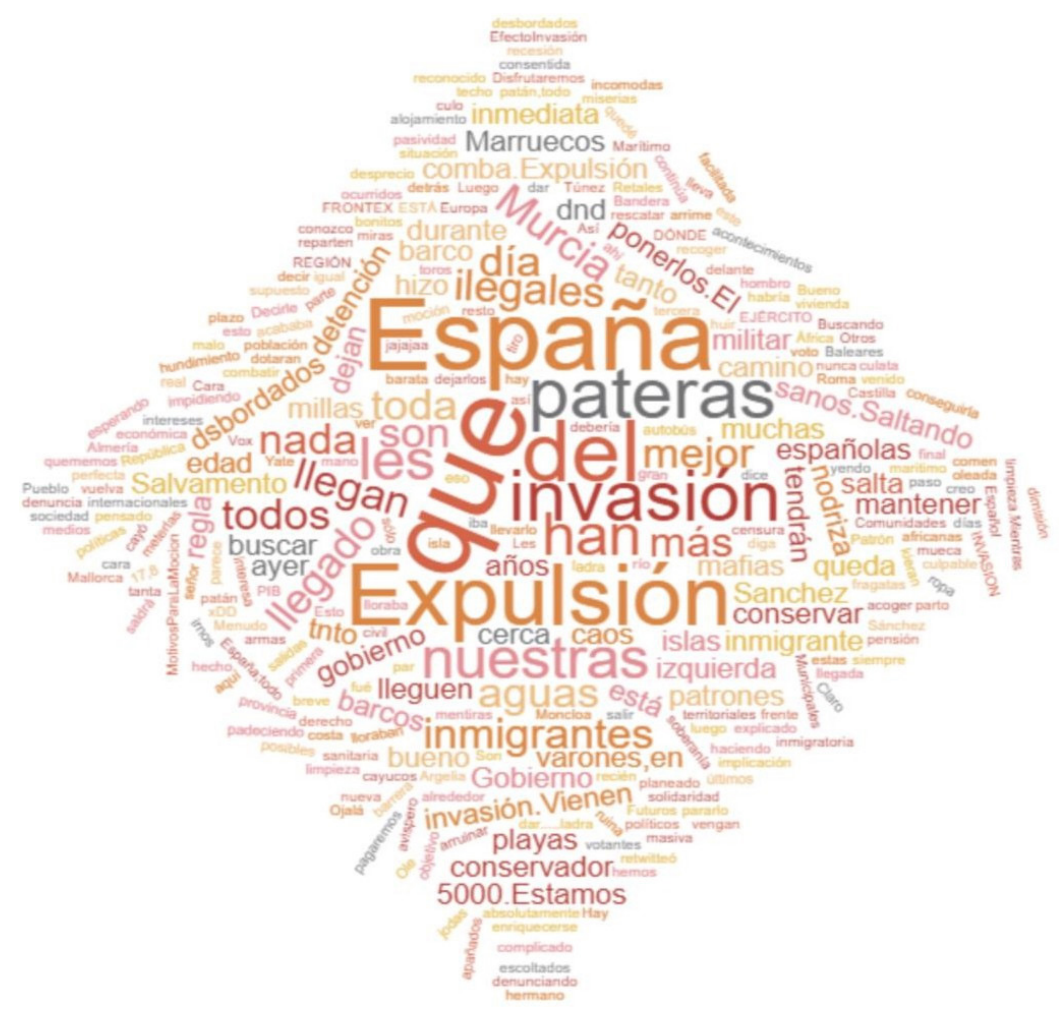

Figura 3. Nube de tags de las respuestas al tuit de la cuenta oficial de Vox. Fuente: elaboración propia.

Partiendo de esta propuesta, podríamos proponer a los estudiantes que reflexionen sobre algunas cuestiones importantes:

- ¿Qué imagen tiene el partido Vox de los inmigrantes?

- ¿Estás de acuerdo con el término “invasión” para describir la llegada de migrantes a España? ¿Piensas lo mismo de los españoles que van a trabajar a otros países, como Reino Unido o Alemania?

- Revisa el hilo de Twitter y piensa en tres argumentos para rebatir alguna de las respuestas

- En el mismo hilo, trata de localizar alguna noticia falsa difundida por los tuiteros

Otro ejemplo adecuado y de permanente actualidad para trabajar en clase es el tema de los refugiados, que puede ser abordado desde diferentes perspectivas. También encontramos ejemplos valiosos en las noticias que se hacen eco de incidentes xenófobos perpetrados por jóvenes o adolescentes. Seguidamente, proponemos algunas opciones que pueden resultar estimulantes para concienciar al alumnado.

Evidentemente, las redes sociales no son axiológicamente negativas, pues en Twitter podemos hallar también expresiones de condena y rechazo de las conductas de discriminación, y de denuncia de las injusticias sociales que dan lugar a noticias terribles como la muerte de un niño refugiado en el mar. Ese enfoque positivo sobre los inmigrantes está presente en noticias como la que publicó el diario La Vanguardia (3/9/2015): "Las redes sociales homenajean a Aylan Kurdi. Dibujos y mensajes recuerdan al niño de tres años encontrado muerto en la turística playa de Turquía”. Aquí, la información va más allá del relato del suceso y se centra en las reacciones de homenaje en la red. Estos materiales también pueden ser útiles para su análisis en clase.

También podemos proponer a los estudiantes el análisis descontextualizado de una fotografía en la que se aprecie un comportamiento reprobable (como la imagen de la figura 5, en la que vemos a una reportera húngara 
zancadilleando a un refugiado y su hijo para obtener una grabación de ellos en el suelo) que sirva para iniciar un debate sobre los límites de la información sobre temas sociales en la "sociedad del espectáculo" (Debord, 1999).

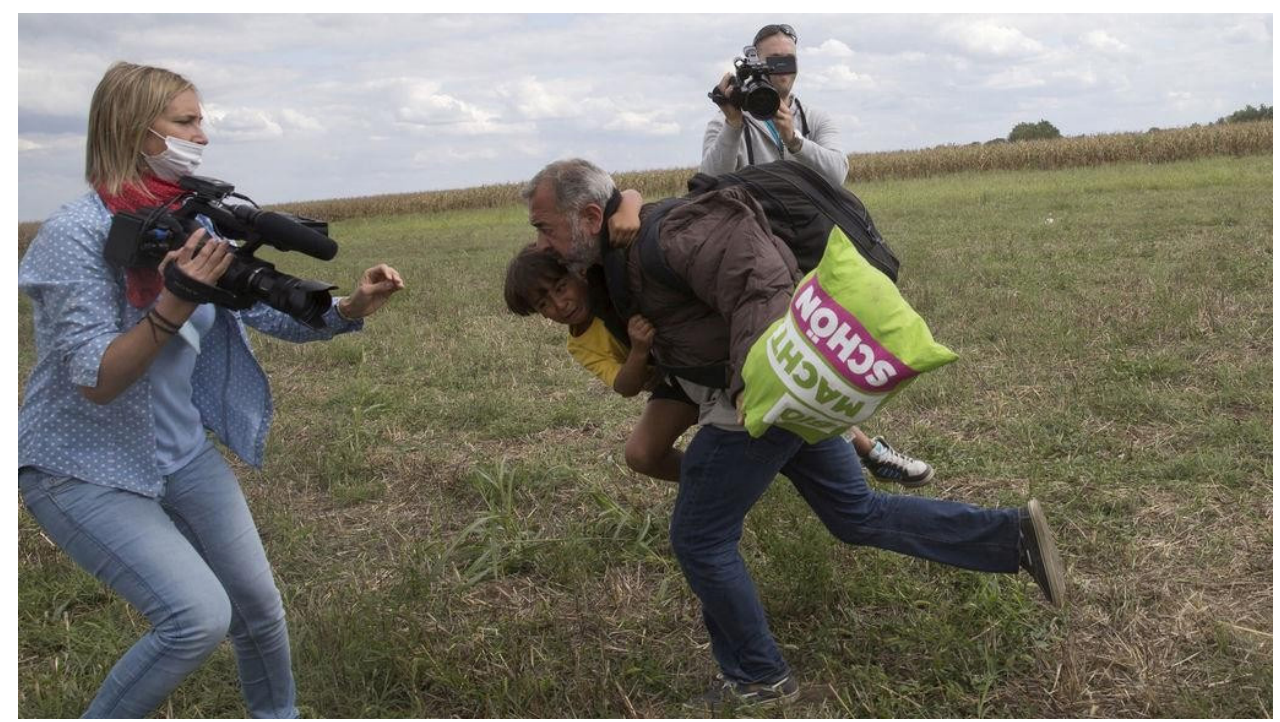

Figura 4. La reportera húngara zancadillea a un refugiado. Fuente: Reuters.

Es básico abordar problemas reales del modo más directo posible, ya que las consignas discriminatorias se quedan en lo superficial. Consideremos, por ejemplo, el tuit del periodista Íñigo Sáenz de Ugarte, que puede servirnos para que los adolescentes reaccionen a esa entrada de Twitter para expresar libremente sus opiniones.

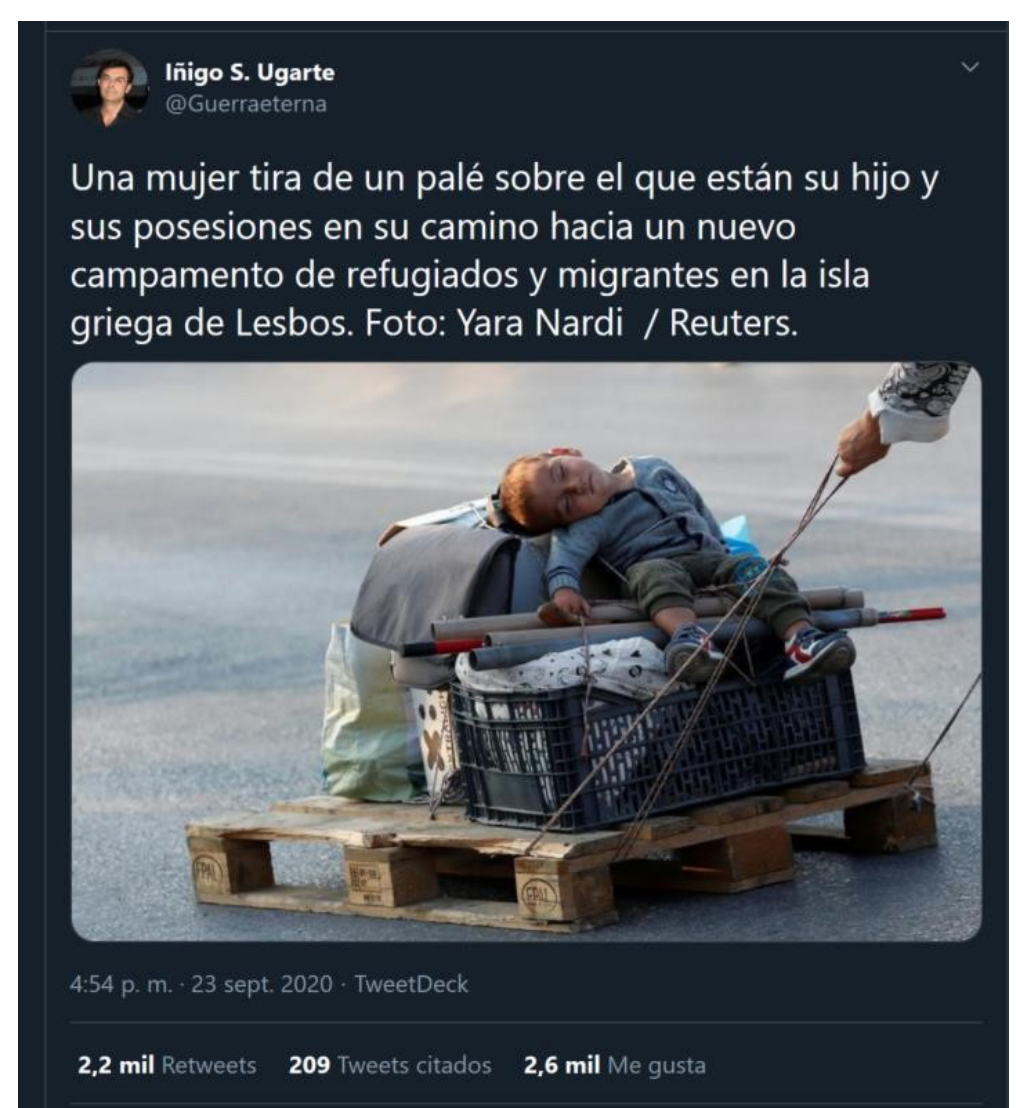

Figura 5. Tuit de Íñigo Sáenz de Ugarte. Fuente: captura de pantalla de la red social Twitter.

Por último, nos hemos fijado en una noticia que pone el foco de atención en la conducta claramente xenófoba de tres adolescentes. Una auténtica educación en valores, centrada en la dimensión ética de la educación (Corbella y Úcar, 2019) tiene que apoyarse en la empatía como pilar fundamental, para lo cual puede resultar operativo que los 
estudiantes se vean reflejados en el espejo que esta noticia nos brinda, a fin de detectar en su propia conducta algunos comportamientos que entrañen algún tipo de prejuicio hacia los extranjeros. Proponemos el análisis de dos imágenes (figura 6) y el planteamiento de las siguientes tareas:

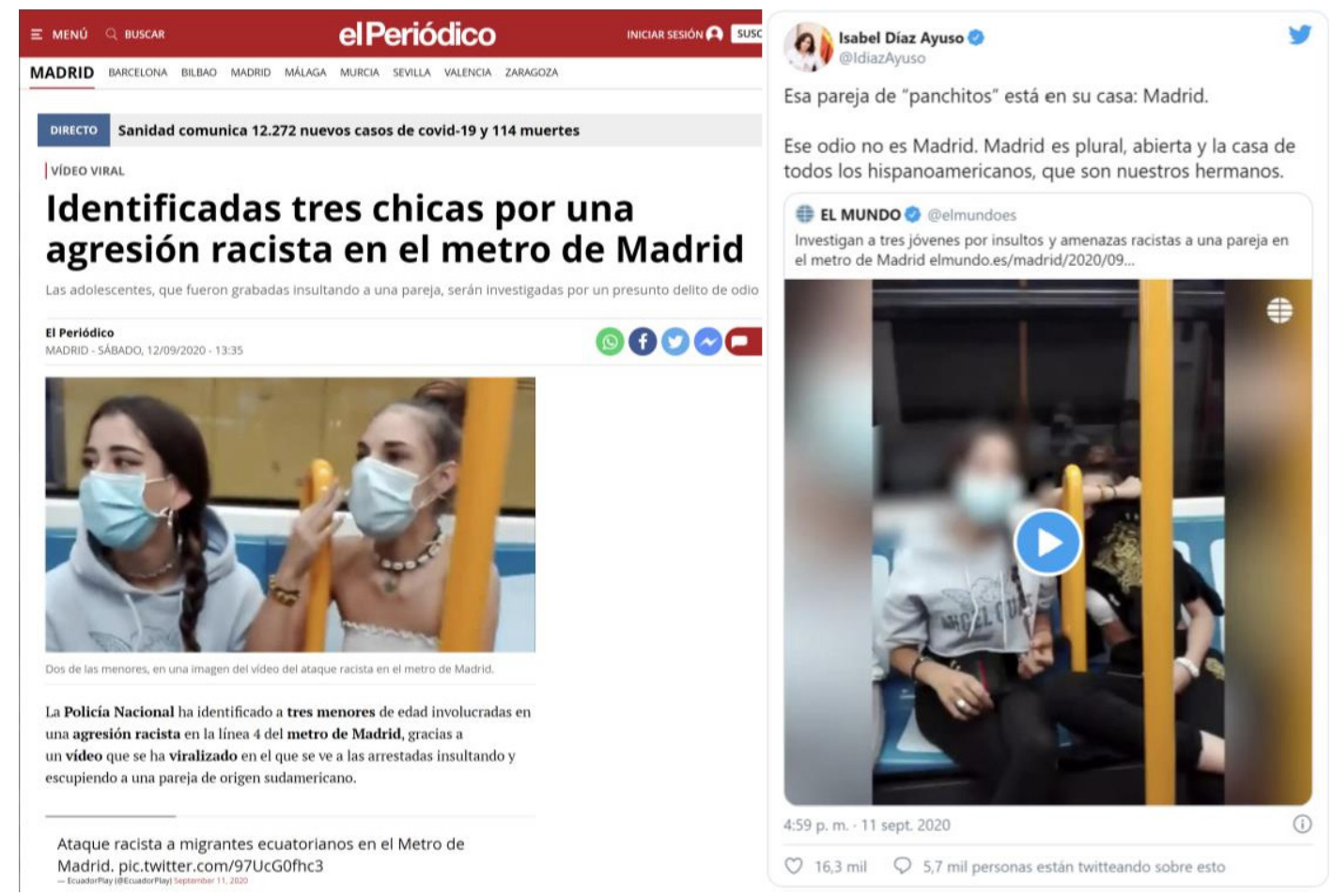

Figura 6. Captura de pantalla de noticia en El Periódico (12/09/2020) y reacción en Twitter de la presidenta de la Comunidad de Madrid. Fuente: El Periódico y red social Twitter

- Reconstruyamos la información buscando datos en Twitter. Por ejemplo, el que aparece referenciado en la noticia y sus reacciones

- Busca en redes mensajes de apoyo a las chicas. ¿Qué clase de argumentos esgrimen?

- Una de las chicas implicadas en el ataque racista responde en redes a la polémica: "Lo que dije en el metro es mi puta opinión y no me la vais a quitar, ¿vale? Porque lo sigo opinando y, ¡ya está! Y este es mi país. Y yo estoy en mi país. ¿Racista? ¡Mucho! ¡Muchísimo!”. ¿Por qué crees que vincula racismo con “ser de un país”?

- Imagina que eres uno de los migrantes atacados y lees uno de esos mensajes en Twitter. ¿Qué responderías?

- Debate: La respuesta de apoyo de la Presidenta de la Comunidad de Madrid a los migrantes agredidos fue criticada por algunas personas en Twitter. ¿A qué crees que se debe?

\section{CONCLUSIONES}

Con este artículo hemos intentado mostrar el progresivo auge de los mensajes populistas de rechazo a los extranjeros y, en general, de odio a lo diferente en redes sociales como Twitter, fenómeno que obliga a los docentes a tomar conciencia del problema e incorporar prácticas de trabajo grupal en el aula encaminadas a analizar y debatir sobre este discurso insolidario y excluyente, a fin de educar en valores "a tiempo", atendiendo a la realidad del presente (Caride, 2020). Lo verdaderamente preocupante es que la palabra induce a la acción, y cada vez son más numerosos los delitos de odio cometidos contra personas extranjeras en España.

El desarrollo de redes sociales como Twitter ha traído consigo una transformación de los patrones de interacción, lo cual exige comprender la lógica de funcionamiento de los mecanismos comunicativos derivados de la Web 2.0. En paralelo, asistimos al crecimiento imparable de los mensajes de odio en Twitter, siendo especialmente alarmante entre los menores, mucho más permeables a la manipulación, pues aún están indefensos ante los peligros que se ocultan tras los nuevos escenarios virtuales.

Por todo lo expuesto, urge implementar en el aula estrategias orientadas a la educación en valores y la sensibilización sobre la tolerancia y la inclusión sociocultural. Cualquier tratamiento de cuestiones ideológicas en clase es problemático, por lo que, naturalmente, el posicionamiento crítico en el aula que defendemos ha de estar desprovisto de sesgos partidistas, para enmarcarse en una formación cívica integral de los estudiantes. No basta con abordar en el aula el concepto de racismo y, en paralelo, repasar en clase de Lengua castellana y Literatura los géneros periodísticos tradicionales: se trata de enseñar a los adolescentes, que se encuentran inmersos de lleno en la construcción de su 
propia identidad y del concepto de alteridad, a "leer" la prensa, aprovechando las herramientas que ellos mismos utilizan para informarse, de modo que sean capaces de detectar los mensajes populistas, y valorarlos críticamente. Evidentemente, ello conlleva un esfuerzo de los docentes para actualizar sus métodos de enseñanza, procurando motivar a los discentes de un modo atractivo y participativo. Precisamente, estimamos relevante profundizar en próximos trabajos en la percepción de la utilidad de propuestas didácticas como las apuntadas aquí por parte del profesorado. Sabemos que los adolescentes quieren ser parte activa de su proceso de enseñanza-aprendizaje, por lo que no tiene sentido recurrir a las tradicionales clases teóricas magistrales; al contrario, para poder hacerles partícipes de dicho proceso, y como señala Prensky (2013), proporcionar "una educación conectada con la realidad, con su realidad" deberemos utilizar las herramientas online que, como nativos digitales, manejan a diario.

\section{BIBLIOGRAFÍA}

Alonso Alonso, Rosario (1997) El racismo y la xenofobia como objeto de intervención social con jóvenes. Servicios Sociales y Política Social, 37, 33-45.

Calvo Buezas, José Luis (2012). Las actitudes de xenofobia latente en los jóvenes extremeños. Almenara: Revista Extremeña de Ciencias Sociales, 4. Https://sites.google.com/site/almenararevistasociologia/home/almenara-no-4---primersemestre-2012/articulos-de-investigacion-almenara-no-4/lasactitudesdexenofobialatenteenlosjovenesextremenos.

Calvo Buezas, José Luis (2005). Inmigración y racismo. La educación ética en valores solidarios. Sociedad y Utopia: Revista de Ciencias Sociales, 25, 197-214.

Caride, José Antonio (2020). Educar y educarnos a tiempo, pedagógica y socialmente. Revista Española de Pedagogía, 78(277), 395-413. Https://doi.org/10.22550/REP78-3-2020-03.

Castells, Manel (1999). La era de la información. Madrid: Alianza Editorial.

Cereceda Fernández-Oruña, Jaime y otros (2019). Informe de la evolución de los delitos de odio en España. Madrid: Ministerio del Interior. Http://www.interior.gob.es/documents/642012/3479677/Informe+sobre+la+evoluci\%C3\%B $3 \mathrm{n}+$ de + delitos + de + odio + en + Espa $\% C 3 \% B 1 a \% 2 C \% 20 a \% C 3 \% B 1 o+2019 / 344089$ ef-15e6-4a7b-8925-f2b64c117a0a.

Cobano-Delgado, Verónica y Mercedes Llorent-Vaquero (2017). Identidad cultural del alumnado marroquí: estado de la cuestión en los centros de educación secundaria públicos de Andalucía. Pedagogía Social. Revista Interuniversitaria, 29, 81-96. Https://doi.org/10.7179/PSRI_2017.29.06.

Corbella, Laura y Xavier Úcar (2019). The ethical dimensión and values involved in social education. Perspectives of scholars, educators and parents. Ramon Lull Journal of Applied Ethics, 10, 91-122.

Debord, Guy (1999). La sociedad del espectáculo. Valencia: Pretextos.

Durán, Saray, Natalia Sueiro y Sandra García (2018). Ciberespect, cultivando una paisaxe dixital de respecto. Revista Galega de Traballo Social, 21, 123-142.

Escalona Velázquez, Alejandro (2012). La identidad: camino hacia la individualidad cultural. Revista Adicción y Ciencia, 2(1), 1-6.

Federación SOS Racismo (2019). Informe anual 2018 sobre el racismo en el Estado Español. Https://sosracismo.eu/tag/ informe/

Fernández Huerta, Christian (2015). Participación juvenil y activismo digital. Una introducción a una nueva agenda de estudio. TecCom Studies: Estudios de Tecnología y Comunicación, 6, 7-18.

García Martínez, Alfonso (2005). La educación intercultural y las nuevas lógicas del racismo. En Rafael Calvo de León (coord.). Los jóvenes, el conflicto y la pluriculturalidad: actuaciones desde la educación social (pp. 163-178). Burgos: Diputación Provincial de Burgos.

Hernández Fernández, Coral, José Antonio Alcobea Hernando y María Cadilla Baz (2014). La percepción del riesgo en las redes sociales entre la juventud inmigrada en España. RAE-IC: Revista de la Asociación Española de Investigación de la Comunicación, 1(1), 2-11.

Jiménez Bautista, Francisco (2005). "Imagen y percepción de los jóvenes de Granada sobre la inmigración marroquí. Convergencia. Revista de Ciencias Sociales, 39, 183-216.

Jiménez Bautista, Francisco (2006). La inmigración marroquí en Granada: su imagen y percepción por los jóvenes granadinos. Estudios Geográficos, 67(261), 549-578.

Jiménez Mañas, Mar (2012). El reto de la ética como compromiso educativo. En Remedios Sánchez García (ed.). Compromiso docente y realidad educativa. Retos para el maestro del siglo XXI (pp. 93-101). Madrid: Síntesis.

Machado-Casas, Margarita, Iliana Alanis y Elisa Ruiz (2017). Innovative technologies as social pedagogy: transforming informal educational practices in the United States. Pedagogía Social. Revista Interuniversitaria, 29, 55-66. Https:// doi.org/10.7179/PSRI_2017.29.04.

Mancera, Ana y Ana Pano (2013). El discurso político en Twitter. Análisis de mensajes que “trinan”. Barcelona: Anthropos.

Martínez Ezquerro, Aurora (2016). Las TIC en lengua castellana y literatura. Criterios de calidad y recursos didácticos. Revista DIM: Didáctica, Innovación y Multimedia, 34.

Martínez-Usarralde, María Jesús, Carmen Lloret-Catalá y Manuel Céspedes (2017). Lo que hacen las mejores escuelas integradoras de alumnado inmigrante: indicadores de buenas prácticas. Pedagogía Social. Revista Interuniversitaria, 29, 41-54. Https://doi.org/10.7179/PSRI_2017.29.03. 
Mata Benito, Patricia y Belén Ballesteros Velázquez (2008). El racismo desde la perspectiva de los adolescentes: su significado y experiencias. Revista Europea de Etnografía de la Educación, 5, 117-130.

Olmos Alcaraz, Antonia (coord.) (2020): Jóvenes, redes sociales virtuales y nuevas lógicas de funcionamiento del racismo: Etnografía virtual sobre representaciones y discursos de alteridad e identidad. Madrid: Centro Reina Sofía Sobre Adolescencia y Juventud - FAD. Doi: 10.5281/zenodo.3666178.

Peiró i Grègori, Salvador y Gladys Merma Molina (2012). La interculturalidad en la educación. Situación y fundamentos de la educación intercultural basada en valores. Barataria. Revista Castellano-Manchega de Ciencias Sociales, 12 , 127-139.

Prensky, Marc (2001). Digital natives, digital immigrants. On the Horizon, 9(5), 1-6. Http://www.marcprensky.com/ writing/Prensky\%20-\%20Digital\%20Natives,\%20Digital\%20Immigrants\%20-\%20Part1.pdf

Prensky, Marc (2013). Enseñar a nativos digitales. Madrid: SM.

Puig Rovira, Josep María y Xus Martín García (1998). La educación moral en la escuela. Teoría y práctica. Barcelona: Edebé.

Sánchez Moral, Eva María y César Vallejo Martín-Albo (2003). La educación intercultural y la educación en valores. Educación y Futuro: Revista de Investigación Aplicada y Experiencias Educativas, 8, 71-80.

Terrón-Caro, Teresa, Rocío Cárdenas-Rodríguez y Rocío Rodríguez (2017). Educación intercultural inclusiva. Funciones de los/as educadores/as sociales en instituciones educativas. Pedagogía Social. Revista Interuniversitaria, 29 , 25-40. Https://doi.org/10.7179/PSRI_2017.29.02.

Tudela Canaviri, Patricia, Juan Carlos Ballesteros Guerra, Ana Rubio Castillo y Anna Sanmartín Ortí (2020). Barómetro juvenil, 2019. Discriminación y tolerancia hacia la diversidad. Madrid: Centro Reina Sofía sobre Adolescencia y Juventud - FAD. Doi: 10.5281/zenodo.3600993.

Van Dijk, Teun A. (1997): Racismo y análisis crítico de los medios. Barcelona: Paidós. 